\title{
Propiedades psicométricas de la Escala de Enriquecimiento Trabajo-Familia para la población argentina
}

\section{Psychometric Properties of the Work-Family Enrichment Scale for the Argentinian Population}

\author{
Alicia Omar a,b, Florencia Urteaga ${ }^{\mathrm{c}}$, \& Solana Salessi ${ }^{\mathrm{a}, \mathrm{d}}$ \\ ${ }^{a}$ Consejo Nacional de Investigaciones Científicas y Técnicas, Conicet, Argentina \\ ${ }^{b}$ Universidad Nacional de Rosario, Rosario, Argentina \\ ${ }^{c}$ Universidad Nacional de Entre Ríos, Paraná, Argentina \\ ${ }^{d}$ Pontificia Universidad Católica Argentina, Rosario, Argentina
}

\begin{abstract}
Resumen: El objetivo del presente estudio fue analizar las propiedades psicométricas de la Escala de Enriquecimiento Trabajo-Familia (EETF) de Carlson, la que ha sido desarrollada para medir la interacción positiva entre ambos dominios vitales (desde el trabajo a la familia y desde la familia al trabajo). Se ejecutó un estudio piloto con 120 trabajadores argentinos para analizar la equivalencia conceptual, semántica y operacional de la escala. Se elaboró un estudio principal a efectos de analizar las propiedades psicométricas de la escala puesta a punto en el estudio piloto; en esta ocasión se trabajó sobre una nueva muestra integrada por 198 trabajadores argentinos, con edades entre 20 y 51 años que tenían, al menos, un hijo o un familiar mayor a su cargo. El análisis factorial exploratorio indicó una estructura bifactorial, la que quedó corroborada a través de análisis factoriales confirmatorios. Los dos factores del constructo mostraron adecuada validez convergente, buenos índices de confiabilidad alfa ordinal e invarianza configural y métrica entre varones y mujeres. A partir de los resultados obtenidos, tanto académicos, científicos organizacionales y gerentes de recursos humanos dispondrán de un instrumento válido y confiable para medir el enriquecimiento trabajo-familia entre los trabajadores argentinos.
\end{abstract}

Palabras clave: enriquecimiento trabajo-familia, escala, propiedades psicométricas, trabajadores adultos.
Abstract: The aim of this study was to analyze the psychometric properties of the Work-Family Enrichment scale by Carlson, which has been developed to measure the positive interaction between these two life domains (from work to family and from family to work). A pilot study with 120 Argentinian workers was performed to analyze the conceptual, semantic and operational equivalence of the scale. A main study was carried out, in order to analyze the psychometric properties of the scale adapted for the pilot study. The sample comprised 198 Argentinian workers, aged from 20 to 51, who had at least one child or an elderly relative under their care. Exploratory factor analysis indicated a bifactorial structure, which was verified by confirmatory factor analyses. Both dimensions of the construct showed adequate convergent validity, high levels of ordinal alpha reliability, and configural and metric invariance between men and women. The results obtained will provide scholars, organizational scientists and human resource managers with a valid and reliable instrument to measure work-family enrichment among Argentinian workers.

Keywords: work-family enrichment, scale, psychometric properties, adult workers.

Este trabajo ha sido realizado en el marco del subsidio PIP 112-201101000001 otorgado por el Consejo Nacional de Investigaciones Científicas y Técnicas (Conicet), Argentina.

Contacto: A. Omar. Italia 1365 (2000), Rosario, Argentina. Correo electrónico: agraomar@yahoo.com

Cómo citar: Omar, A., Urteaga, F., \& Salessi, S. (2015). Propiedades psicométricas de la Escala de Enriquecimiento Trabajo-Familia para la población argentina. Revista de Psicología, 24(2), 1-18.

http://dx.doi.org/10.5354/0719-0581.2015.37689 


\section{Introducción}

Trabajo y familia son las esferas más importantes en la vida de las personas. Frente al creciente número de familias monoparentales y de parejas en las que ambos miembros trabajan fuera del hogar, el análisis de la interfaz trabajofamilia se ha transformado en un tema de interés central en las sociedades modernas. En línea con el desarrollo de la psicología organizacional positiva (Luthans \& Youssef, 2007), la tendencia actual es expandir el paradigma trabajofamilia de manera que además del lado negativo, incluya también su faceta positiva. Este lado positivo, denominado perspectiva del enriquecimiento trabajofamilia (Greenhaus \& Powell, 2006), supone que combinar los roles laborales y familiares produce efectos positivos que se irradian a todos los dominios de la vida.

El concepto de Enriquecimiento Trabajo-Familia (ETF) asienta sus bases sobre dos grandes pilares teóricos. Por un lado, la teoría de la expansión de recursos (Marks, 1977), que postula que los sujetos pueden generar la energía suficiente como para efectuar los múltiples roles confiados lo que, en lugar de producir tensiones, redunda en mayor bienestar subjetivo. Y, por otro, la teoría de la identidad social (Tajfel \& Turner, 2004) que sostiene que el desempeño de cada rol refuerza la autoidentidad y añade sentido a la vida. Con base en estos marcos teóricos, Greenhaus y Powell (2006) desarrollaron el concepto ETF al que definieron como bidimensional, ya que postularon que los recursos ganados en el trabajo contribuyen a mejorar la calidad de vida familiar, y que sucede lo mismo a la inversa: los recursos ganados en la familia mejoran el rendimiento laboral. Definieron los recursos (habilidades, capacidades físicas y psicológicas, capital social) como "activos que pueden disponerse cuando hay necesidad de solucionar un problema o enfrentar una situación desafiante" (Greenhaus \& Powell, 2006, p. 80), y señalaron que la transferencia de los mismos desde un rol a otro se puede producir de modo directo (vía instrumental), o bien, indirecto (vía emocional).

La investigación previa acerca de la sinergia positiva entre el trabajo y la familia ha empleado constructos tales como derrame positivo, facilitación o mejoramiento (Wayne, Grzywacz, Carlson, \& Kacmar, 2007). Sin embargo, dado su carácter bidireccional, el concepto de ETF no se asimila a ninguno de aquellos constructos previos, desde el momento que supone que el trabajo contribuye a mejorar la calidad de vida familiar (Allis \& O'Driscoll, 2008), y que la participación en la familia mejora el rendimiento en el trabajo (van Steenbergen \& Ellemers, 2009). El modelo del dominio específico (Frone, 2003) ha sido crucial en este sentido, porque ha brindado el andamiaje teórico-conceptual necesario para sostener tales supuestos. Este modelo señala que las facetas bidireccionales del ETF son empíricamente diferentes y presentan patrones exclusivos para cada rol (Byron, 2005), habida cuenta de que los antecedentes se generan en un dominio (rol originario) y sus consecuencias se hacen sentir sobre otro dominio (rol receptivo). A pesar de las posibles implicancias acerca del bienestar del trabajador y de la eficiencia de la organización, hasta ahora esta perspectiva del enriquecimiento ha recibido escasa atención, motivo por el que está demandando mayor número de estudios sistemáticos. 


\section{La operacionalización del constructo enriquecimiento trabajo-familia}

Desde el surgimiento del constructo ETF se han registrado algunos intentos por operacionalizarlo, básicamente, a través de escalas o cuestionarios autodescriptivos. Grzywacz y Marks (2000) elaboraron un cuestionario destinado a medir las interrelaciones entre el trabajo y la familia. Aunque su mayor debilidad ha sido el solapamiento de algunos de sus ítems con los constructos de fatiga y de apoyo familiar. Más tarde, Wayne, Musisca y Fleeson (2004) desarrollaron una escala para medir el lado positivo de la interfaz trabajo-familia. Sin embargo, su escala mide facilitación, más que ETF. Más recientemente, Carlson, Kacmar, Wayne y Grzywacz (2006) diseñaron la Escala de Enriquecimiento Trabajo-Familia (EETF), que explora las dos direcciones del enriquecimiento (desde el trabajo a la familia y desde la familia al trabajo), a través de 18 ítems, con formato Likert de 5 puntos $(1=$ Nunca $; 5$ = Siempre).

La EETF mide el enriquecimiento en la dirección trabajo $\rightarrow$ familia con nueve ítems que comienzan con la expresión "Mi participación en el trabajo" y explora las siguientes tres modalidades: enriquecimiento basado en el capital, que se produce cuando la participación en el trabajo promueve recursos psicosociales (e.g., la confianza) que ayudan al individuo a ser un mejor miembro de la familia (e.g., "Me da un sentido de logro y esto me ayuda a ser un mejor miembro de mi familia"); enriquecimiento basado en la afectividad, que ocurre cuando la participación en el trabajo genera un estado emocional positivo que ayuda al individuo a ser un mejor miembro de la familia (e.g., "Me hace sentir feliz y esto me ayuda a ser un mejor miembro de mi familia"); y enriquecimiento basado en el desarrollo que se produce cuando la participación en el trabajo conduce a la adquisición $o$ al perfeccionamiento de las habilidades, conocimientos o comportamientos que ayudan a una persona a ser un mejor miembro de la familia (e.g., "Me ayuda a entender diferentes puntos de vista y esto a ser un mejor miembro de mi familia").

La EETF también captura el enriquecimiento familia $\rightarrow$ trabajo a través de nueve ítems precedidos por la expresión "Mi participación en la familia". Esta segunda dimensión mide tres formas de enriquecimiento familia $\rightarrow$ trabajo, dos de las cuales (el enriquecimiento basado en el desarrollo y el enriquecimiento basado en la afectividad) son equivalentes a las dimensiones homónimas del enriquecimiento en la dirección trabajo $\rightarrow$ familia, en el sentido de que la involucración en la familia ayuda a la persona a ser un mejor trabajador, y una tercera modalidad, rotulada como eficiencia, que ocurre cuando la participación en la familia proporciona un sentido de responsabilidad y urgencia que ayuda al individuo a ser un mejor trabajador (e.g., "Me lleva a no perder tiempo en mi trabajo y esto me ayuda a ser un mejor trabajador"). La EETF ha sido validada para su empleo con muestras eslovenas (Tement, Korunka, \& Pfifer, 2010); italianas (Ghislieri, Martini, Gatti, \& Colombo, 2011); portuguesas (Vieira, Lopez, \& Matos, 2014) y coreanas (Lim, Choi, \& Song, 2012), entre otras. En términos generales, todas las validaciones han demostrado la bidimensionalidad de la escala y sus adecuados niveles de confiabilidad.

\section{La red nomológica del enriquecimiento}

La investigación realizada en los últimos cinco años, ha contribuido a conformar teórica y empíricamente la red nomológi- 
ca del ETF (McNall, Nicklin, \& Masuda, 2010). Entre los antecedentes ya establecidos, aplicables en ambas direcciones del enriquecimiento, se destacan el bienestar subjetivo, las demandas, la participación y el control sobre los horarios. El bienestar subjetivo ha sido explorado tanto por Hanson, Hammer y Colton (2006) como por Haar y Bardoel (2008). Respecto de las demandas (definidas como la percepción global del grado y la intensidad de la responsabilidad en el trabajo o en la familia), estas han sido exploradas por Demerouti, Peeters y van der Heijden (2012). En relación con la participación (definida como el grado en que el rol en el trabajo (o en la familia) es fundamental para la identidad, sobre todo por el tiempo y el esfuerzo dedicado a cada rol) ha sido estudiada por Meeuwisse, Born y Severiens (2011). Por último, el control del horario de trabajo se refiere al grado en el que los trabajadores son capaces de administrar el horario de inicio y finalización de su trabajo, y su tiempo libre, en tanto que el control del horario en el hogar corresponde al grado en que los trabajadores sienten que controlan el tiempo que le dedican a las tareas del hogar y al momento en que las realizan. Ambos tipos de controles horarios han sido analizados por Carlson, Ferguson, Kacmar, Grzywacz y Whitten (2011) y por McNall et al. (2010). En cuanto a las consecuencias del ETF, investigaciones previas han demostrado que el compromiso (Hakanen, Peeters, \& Perhoniemi, 2011); la satisfacción (Masuda, McNall, Allen, \& Nicklin, 2012; McNall et al., 2010), y el rendimiento (Odle-Dusseau, Britt, \& GreeneShortridge, 2012), suelen ser el resultado del enriquecimiento en uno u otro sentido.

Como hasta el momento no se dispone de la versión adaptada y validada para su empleo con trabajadores argentinos, el objetivo del presente trabajo fue validar la EETF de Carlson et al. (2006), analizando su estructura factorial, confiabilidad, validez e invarianza en función del género. Respecto de la estructura factorial de la escala, se esperó confirmar la bidimensionalidad informada por Carlson et al. (2006). Asimismo, se esperó obtener índices de confiabilidad y validez aceptables tanto para la escala global, como para las subescalas que la integran. Finalmente, se esperó que la escala mostrara invarianza en función del género, por lo que se planteó la siguiente hipótesis: la estructura factorial de la escala de EETF se mantendrá invariante en varones y mujeres.

Disponer de una herramienta con adecuadas propiedades psicométricas para medir el ETF, que además sea breve y parsimoniosa como lo es la EETF, no solo facilitaría la implementación de prácticas e intervenciones concretas en los ámbitos laborales-industriales, sino que también contribuiría a la ejecución de nuevas investigaciones en el área de la psicología organizacional y de la salud. Posiblemente, también promovería nuevas adaptaciones en países de la región, lo que favorecería la validación transcultural del instrumento y la puesta en marcha de estudios comparativos a efectos de lograr un conocimiento más acabado de la interfaz trabajo-familia en el cono sur latinoamericano.

\section{Método}

\section{Diseño}

La presente investigación se inscribe en la categoría de estudios instrumentales (Montero \& León, 2007), ya que se orienta a la adaptación y análisis de las propiedades psicométricas de un instrumento. Con tal propósito se efectuó un estudio piloto y uno principal. 


\section{Estudio piloto}

El objetivo de este estudio fue traducir al español y adaptar la Escala de Enriquecimiento Trabajo-Familia de Carlson para su empleo con muestras argentinas. En esta instancia se realizó el análisis de la equivalencia conceptual, semántica y operacional del instrumento (Herdman, Fox-Rushby, \& Badía, 1998).

La equivalencia conceptual se refiere a la búsqueda de correspondencias de cada concepto entre la cultura donde fue desarrollado el instrumento y la cultura objetivo (en la que se aplicará el instrumento). En esta oportunidad, se efectuó una revisión bibliográfica acerca del tema trabajofamilia, tanto en la cultura original de la escala (Estados Unidos de Norteamérica) como en la argentina. De particular utilidad en esta etapa fue la revisión internacional realizada por Poelmans, O'Driscoll y Beham (2005), la que indica que la interacción positiva entre la familia y el trabajo se asocia con el bienestar subjetivo y la satisfacción laboral en todas las culturas. Simultáneamente, los ítems fueron sometidos a revisión crítica por parte de dos psicólogos organizacionales.

La equivalencia semántica consiste en la traducción de los ítems que conforman el instrumento, conservando el significado entre idiomas diferentes. En este caso, la traducción-retraducción de los ítems fue realizada por dos traductores de inglés. $\mathrm{La}$ equivalencia operacional se refiere al mantenimiento de las características operativas antes y durante la aplicación de los instrumentos, básicamente en lo referente al tiempo que demanda completarlo, la claridad de las instrucciones para efectuar la tarea y la adecuación semántica y sintáctica de los ítems. En esta ocasión, la versión prototípica traducida al español de la escala autoadministrable de Carlson presentada bajo un formato Likert de cinco puntos (variando desde $1=$ Nunca a 5 = Siempre), fue aplicada a una muestra por disponibilidad integrada por $120 \mathrm{em}$ pleados de organizaciones radicadas en la ciudad de Rosario (Argentina). El 64\% de los participantes eran mujeres. La media de edad de la muestra fue de 28,25 años $(D E=3,62)$ y la antigüedad laboral promedio fue de 4,20 años $(D E=3,69)$. De acuerdo con el ramo de actividad de los empleados, el $36 \%$ pertenecía a la industria, el $30 \%$ al comercio, el $22 \%$ a empresas de servicios (bancos, aseguradoras, consultoras) y el $12 \%$ restante a instituciones educativas. La totalidad de la muestra completó la escala en sus respectivos lugares de trabajo y en horarios cedidos por las empresas para fines de capacitación y/o investigación. En todos los casos, una vez concluida la aplicación de la escala, se habilitó un espacio para que los participantes pudieran opinar acerca de la claridad de los ítems, facilidad de comprensión, posibles ambigüedades, tiempo requerido para responderlos y aspectos similares.

\section{Estudio principal}

El objetivo de este estudio fue analizar las propiedades psicométricas de la versión traducida y adaptada de la Escala de Enriquecimiento Trabajo-Familia desarrollada por Carlson et al. (2006).

\section{Participantes}

El tamaño de la muestra se determinó con base en el criterio de contar con un mínimo de 200 casos (Shah \& Goldstein, 2006). Considerando este criterio, y como el presente estudio se focalizó en el enriquecimiento entre el trabajo y la familia de los empleados, se invitó a participar solo a trabajadores que tenían hijos o familiares a cargo. De esta manera, se inte- 
gró una muestra inicial de 210 empleados, de los cuales fueron descartados 12 casos por no haber completado totalmente el protocolo de recolección de datos. La muestra definitiva quedó conformada por 198 empleados (110 varones y 88 mujeres) de organizaciones localizadas en la ciudad de Rosario (Argentina). El promedio de edad de las mujeres fue de 39 años, mientras que el de los varones fue de 35 años. La antigüedad laboral promedio fue de 4,5 años para los empleados de empresas privadas y nueve años para los de empresas públicas. El $73 \%$ de la muestra tenía estudios secundarios completos o universitarios incompletos, en tanto que el $27 \%$ restante tenía formación universitaria y ocupaba puestos gerenciales o de cierta responsabilidad. En cuanto a la actividad de las empresas, 29\% eran fábricas o industrias, $25 \%$ pertenecía al área de servicios (bancos, seguros, provisión de gas o de electricidad), $20 \%$ al comercio, $15 \%$ a educación y $11 \%$ a salud.

\section{Instrumento}

El ETF fue medido con la versión autoadministrable -traducida al español y puesta a punto durante la prueba piloto-, de la Escala de Enriquecimiento TrabajoFamilia de Carlson et al. (2006), integrada por 18 ítems con formato Likert de cinco puntos (variando desde $1=$ Nunca a $5=$ Siempre).

\section{Procedimiento}

La recolección de los datos se efectuó en el seno de aquellas organizaciones que, luego de conocer los objetivos del estudio, aceptaron formar parte de la investigación. En todos los casos, los participantes completaron voluntariamente un cuadernillo preparado para la ocasión, de manera individual o en pequeños grupos, en horarios de trabajo y en los lugares físicos destinados por las autoridades organizacionales para tal propósito. Además de la EETF, cada cuadernillo contenía una primera hoja donde se explicaba el objetivo del estudio, se daban las instrucciones para responder y se aseguraba el anonimato y la confidencialidad de la información suministrada $y$, una segunda hoja, conteniendo un formulario de consentimiento informado.

\section{Análisis de los datos}

Se examinó la matriz de datos con miras a detectar la presencia de casos perdidos. El supuesto de normalidad de los ítems se verificó mediante el cálculo del tamaño de la asimetría y curtosis. Valores de curtosis y asimetría inferiores a 1,60 fueron considerados aceptables (George \& Mallery, 2011). Por la naturaleza ordinal de los datos, se exploró la estructura factorial de la escala por medio de Análisis Factoriales Exploratorios (AFE), usando el método de los mínimos cuadrados no ponderados (ULS, por su nombre en inglés Unweighted Least Squares) basado en una matriz de correlaciones policóricas (Lloret, Ferreres, Hernández, \& Tomás, 2014). Los índices de adecuación muestral fueron evaluados a través de las pruebas de Kaiser-Meyer-Olkin y de esfericidad de Bartlett. Para determinar el número de factores por retener se recurrió a la conjugación de los criterios basados en la regla Kaiser-Gutman de autovalores mayores a $1 \mathrm{y}$ el scree test (tomando en cuenta los componentes ubicados por encima de la curva del gráfico de sedimentación). El criterio para la selección de los ítems fue que pesaran ,40 o más sobre el factor y que no saturaran significativamente sobre dos factores al mismo tiempo (Lloret et al., 2014). Se utilizó la rotación oblicua Promin, porque existía la presunción de que los elementos de la escala estuvieran correlacionados. 
Se examinó y comparó el ajuste del modelo obtenido en el AFE con otros modelos alternativos, a través del cálculo de Análisis Factoriales Confirmatorios (AFC). Se empleó el método de estimación de máxima verosimilitud (ML, por su nombre en inglés Maximum Likelihood) con la corrección robusta de Satorra-Bentler (S-B) (Bentler, 2006; Satorra, 2002), recomendado cuando los datos provienen de escalas ordinales (Bentler, 2006). Para evaluar el ajuste de los modelos se emplearon diversos indicadores. Se utilizó el estadístico Satorra-Bentler $\chi^{2}$, el índice de ajuste comparativo (CFI, por su nombre en inglés Comparative Fit Index) y el error cuadrático medio de aproximación (RMSEA, por su nombre en inglés Root Mean Square Error of Approximation). Los valores utilizados para la bondad de ajuste del modelo fueron los siguientes: índice CFI, valores entre ,90 y ,95 o superiores son considerados como ajustes aceptables a excelentes para el modelo y RMSEA, valores entre ,05 y ,08 (Hu \& Bentler, 1999). Asimismo, se tuvieron en cuenta los coeficientes estandarizados $(\beta)$ de cada variable observada en la variable latente (Hair, Black, Babin, Anderson, \& Tatham, 2006).

La confiabilidad del instrumento se evaluó mediante el estadístico alfa ordinal, diseñado específicamente para el tratamiento de variables categóricas (Gadermann, Guhn, \& Zumbo, 2012). En este caso, valores iguales o superiores a ,70 son considerados evidencia suficiente de confiabilidad (Zumbo \& Oliden, 2008). La validez convergente se verificó mediante el cálculo de la varianza media extraída (AVE, por su nombre en inglés Average Variance Extracted), la que indica que la varianza del constructo se puede explicar a través de los indicadores elegidos (Fornell \& Larcker, 1981). Valores iguales o mayores a ,50 (Bagozzi \& Yi,
1998) son considerados evidencia de validez convergente. Finalmente, se contrastaron los supuestos de invarianza configural y métrica entre varones y mujeres. La invarianza configural hace referencia a la equivalencia de la estructura factorial entre los diferentes grupos. La invarianza métrica atañe al grado en que los parámetros del modelo (en este caso las cargas factoriales) son similares entre los grupos comparados. Para explorar la invarianza configural se efectuó un AFC multigrupo, ajustando el modelo sin restricciones en varones y mujeres. Para determinar la invarianza métrica se realizó la prueba de diferencia de $\chi^{2}$, contrastando el $\chi^{2}$ obtenido en un modelo no constreñido con el $\chi^{2}$ correspondiente a un modelo con restricciones sobre los coeficientes de regresión. Si este valor es estadísticamente significativo, indica que las restricciones especificadas en el modelo más restrictivo no se sostienen; es decir, los dos modelos no son equivalentes entre los grupos (Dimitrov, 2010). El procesamiento y análisis de datos se realizó con los programas SPSS versión 15, Factor versión 9.2 y EQS versión 6.1.

\section{Resultados}

\section{Estudio piloto}

Equivalencia conceptual. La revisión del contenido de cada ítem por parte de los dos psicólogos organizacionales, a la luz de su experiencia profesional y de la bibliografía científica en torno al tema trabajofamilia, indicó que los ítems incluidos en la escala cubrían los dominios del enriquecimiento postulados por Carlson (desde el trabajo a la familia y desde la familia al trabajo), y que la terminología empleada en la redacción de los ítems reflejaba el vocabulario habitual de la población objetivo (trabajadores argentinos). 
Equivalencia semántica. El análisis de la equivalencia semántica fue realizado en tres etapas: el instrumento original fue traducido del inglés al español (argentino); dos traductores de inglés retradujeron la versión argentina al inglés; los mismos traductores compararon a ciegas las dos formas del instrumento a efectos de identificar la concordancia entre el ítem original y el traducido, en función de cuatro niveles de equivalencia: inalterado, poco alterado, bastante alterado y completamente alterado. Ambos profesionales indicaron que se había mantenido inalterada la concordancia entre cada ítem original y el traducido al español, por lo que concluyeron que la versión argentina de la escala de enriquecimiento presentaba adecuada equivalencia semántica.

Equivalencia operacional. Los participantes del estudio piloto señalaron que las instrucciones para realizar la tarea estaban claramente redactadas, que no tuvieron inconvenientes para comprender el contenido de los ítems, y que la escala para responder los ítems (Likert de cinco puntos) no les generaba dificultades.

\section{Estudio principal}

Análisis preliminar de los ítems. La inspección preliminar de los datos mostró que no había casos perdidos, por lo que no era necesario aplicar algún procedimiento de imputación. El tamaño de la asimetría y curtosis demostró el supuesto de normalidad de los ítems y la pertinencia de ejecutar el AFE previsto (Miles \& Shevlin, 2001). Diecisiete de los 18 ítems presentaron valores de asimetría y curtosis entre $+1,00$ y $-1,00$ (tabla 1), considerados muy apropiados por los especialistas (George \& Mallery, 2011).

Tabla 1

Datos descriptivos correspondientes a los ítems de la escala original de ETF

\begin{tabular}{ccccc}
\hline Ítem & Media & $D E$ & Asimetría & Curtosis \\
\hline 1 & 3,39 & 1,18 &,- 46 &,- 52 \\
2 & 3,13 & 1,07 &,- 11 &,- 16 \\
3 & 3,59 & 1,00 &,- 84 &,- 07 \\
4 & 3,28 & 1,04 &,- 19 &,- 28 \\
5 & 3,15 & 1,02 &,- 13 &,- 47 \\
6 & 3,52 & 1,22 &,- 67 &,- 41 \\
7 & 3,14 & 1,14 &,- 45 &,- 29 \\
8 & 3,35 & 1,28 &,- 36 &,- 32 \\
9 & 3,21 & 1,25 & $-1,18$ & $-1,41$ \\
10 & 3,39 & 1,00 &,- 27 &,- 43 \\
11 & 3,42 & 1,13 &,- 22 &,- 32 \\
12 & 3,16 & 1,09 &,- 14 &,- 18 \\
13 & 3,27 & 1,05 &,- 21 &,- 16 \\
14 & 3,35 & 1,12 &,- 46 &,- 23 \\
15 & 3,23 & 1,00 &,- 51 &,- 37 \\
16 & 3,19 & 1,21 &,- 39 &,- 28 \\
17 & 3,48 & 1,07 &,- 23 &,- 17 \\
18 & 3,67 & 1,22 &,- 56 &,- 39 \\
\hline
\end{tabular}


Solo el ítem número nueve presentó índices ligeramente superiores, sin embargo, por no llegar a exceder el límite preestablecido, se evaluaron como valores adecuados.

AFE. El índice de adecuación muestral de Kaiser, Meyer y Olkin fue de ,87 lo que indicó que las correlaciones parciales eran pequeñas, en tanto que la prueba de esfericidad de Bartlett fue estadísticamente significativa $\left(\chi^{2}=\right.$ $57178,00 ; p<, 001)$ por lo que se ejecutó un AFE usando el método de los mínimos cuadrados no ponderados. La regla Kaiser-Gutman de autovalores superiores a 1 determinó la existencia de dos factores que explicaron un 52\% de la variancia de respuesta al test. El gráfico scree test para la magnitud de los autovalores (Cattell, 1966) también indicó la pertinencia de retener dos factores. En función de los criterios indicados, se eliminaron cuatro ítems con pesos factoriales menores a ,40 (Mi participación en el trabajo "Me ayuda a adquirir habilidades y esto me ayuda a ser un mejor miembro de mi familia"; "Me produce alegría y esto me ayuda a ser un mejor miembro de mi familia"; "Me ayuda a sentirme realizado y esto me ayuda a ser un mejor miembro de mi familia"; y Mi participación en mi familia "Me produce alegría y esto me ayuda a ser un mejor trabajador") y dos ítems que pesaron en los dos factores identificados (Mi participación en el trabajo "Me ayuda a aprender cosas nuevas y esto me ayuda a ser un mejor trabajador"; y Mi participación en mi familia "Me hace estar más concentrado en lo que hago y esto me ayuda a ser un mejor trabajador"), por lo que se retuvieron 12 de los 18 reactivos de la escala original. El cálculo de un segundo AFE también con el método de los mínimos cuadros no ponderados, sobre los 12 ítems retenidos, permitió identificar la estructura final de la escala. Nuevamente se identificaron dos factores con autovalores mayores a 1 (figura 1) que, en esta oportunidad, explicaron casi el $60 \%$ de la varianza (tabla 2).

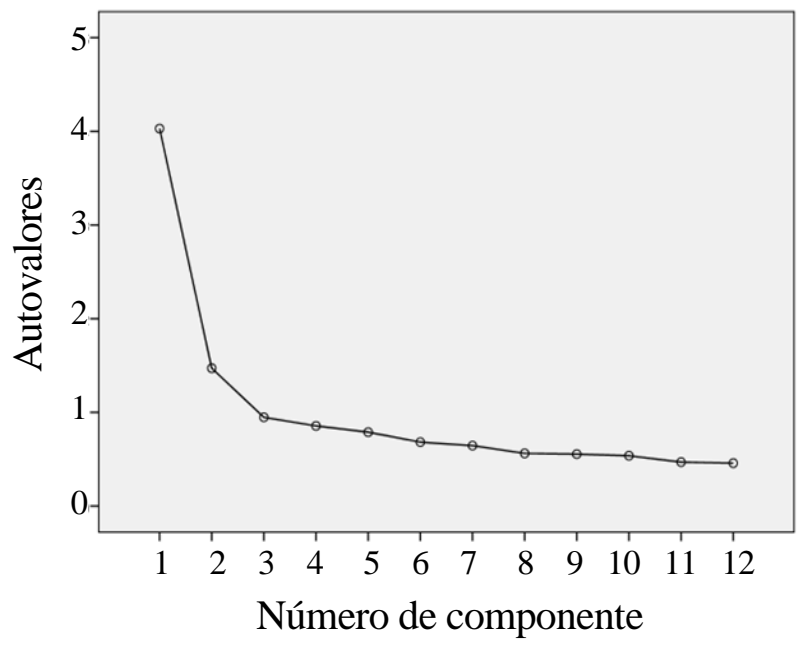

Figura 1. Gráfico de sedimentación correspondiente al AFE de la adaptación de la escala de EETF. 
Para facilitar la interpretación de las cargas factoriales se efectuó una rotación oblicua (Promin), dado que ofrece soluciones más estables y precisas en factores correlacionados. En la tabla 2 se puede apreciar que los 12 ítems presentaron cargas factoriales aceptables (Hinkin, 1998) y todos saturaron en un solo factor, agrupándose en las dos dimensiones propuestas originalmente por los autores de la escala. Tal distribución permitió mantener la denominación propuesta para cada dimensión por Carlson et al. (2006): enriquecimiento trabajo $\rightarrow$ familia $\mathrm{y}$ enriquecimiento familia $\rightarrow$ trabajo.

Tabla 2

Estructura factorial de la adaptación argentina de la EETF de Carlson

\begin{tabular}{|c|c|c|}
\hline & \multicolumn{2}{|c|}{ Factores } \\
\hline & 1 & 2 \\
\hline \multicolumn{3}{|l|}{ Factor 1: Enriquecimiento trabajo $\rightarrow$ familia $(\mathrm{ETF})$} \\
\hline \multicolumn{3}{|l|}{ Mi participación en el trabajo } \\
\hline $\begin{array}{l}\text { 1. Me pone de buen humor y esto me ayuda a ser un mejor miembro } \\
\text { de mi familia (ETF basado en la afectividad) }\end{array}$ & ,798 &, 169 \\
\hline $\begin{array}{l}\text { 2. Me da un sentido de logro y esto me ayuda a ser un mejor miem- } \\
\text { bro de mi familia (ETF basado en el capital) }\end{array}$ & ,705 & ,295 \\
\hline $\begin{array}{l}\text { 3. Me hace sentir feliz y esto me ayuda a ser un mejor miembro de mi } \\
\text { familia (ETF basado en la afectividad) }\end{array}$ & ,688 &, 128 \\
\hline $\begin{array}{l}\text { 4. Me ayuda a entender diferentes puntos de vista y esto me ayuda a } \\
\text { ser un mejor miembro de mi familia (ETF basado en el desarrollo) }\end{array}$ &, 687 &, 155 \\
\hline $\begin{array}{l}\text { 5. Me proporciona una sensación de éxito y esto me ayuda a ser un } \\
\text { mejor miembro de mi familia (ETF basado en el capital) }\end{array}$ & ,619 &, 173 \\
\hline $\begin{array}{l}\text { 6. Me ayuda a adquirir conocimientos y esto me ayuda a ser un mejor } \\
\text { miembro de mi familia (ETF basado en el desarrollo). }\end{array}$ & ,602 & ,196 \\
\hline$(M=3,39 ; D E=1,08)$ & & \\
\hline \multicolumn{3}{|l|}{ Factor 2: Enriquecimiento familia $\rightarrow$ trabajo $(\mathrm{EFT})$} \\
\hline \multicolumn{3}{|l|}{ Mi participación en mi familia } \\
\hline $\begin{array}{l}\text { 7. Me ayuda a usar mejor el tiempo de trabajo y esto me ayuda a ser } \\
\text { un mejor trabajador (EFT basado en la eficacia) }\end{array}$ &, 081 & ,836 \\
\hline $\begin{array}{l}\text { 8. Me obliga a no perder tiempo en el trabajo y esto me ayuda a ser } \\
\text { un mejor trabajador (EFT basado en la eficacia) }\end{array}$ &, 176 &, 775 \\
\hline $\begin{array}{l}\text { 9. Me hace sentir feliz y esto me ayuda a ser un mejor trabajador } \\
\text { (EFT basado en la afectividad) }\end{array}$ & 009 &, 733 \\
\hline $\begin{array}{l}\text { 10. Me pone de buen humor y esto me ayuda a ser un mejor trabaja- } \\
\text { dor (EFT basado en la afectividad) }\end{array}$ & ,085 &, 714 \\
\hline $\begin{array}{l}\text { 11. Me ayuda a adquirir habilidades y esto me ayuda a ser un mejor } \\
\text { trabajador (EFT basado en el desarrollo) }\end{array}$ & , 130 &, 713 \\
\hline $\begin{array}{l}\text { 12. Me ayuda a adquirir conocimientos y esto me ayuda a ser un me- } \\
\text { jor trabajador (EFT basado en el desarrollo) }\end{array}$ & ,204 & ,664 \\
\hline \multicolumn{3}{|l|}{$(M=3,15 ; D E=1,01)$} \\
\hline Varianza explicada & 32,25 & 27,50 \\
\hline
\end{tabular}


AFC. El modelo derivado del AFE, integrado por dos factores latentes correlacionados con 12 ítems como indicadores observables y sus respectivos errores de medición, se comparó con dos modelos alternativos a través de AFC. Los modelos alternativos fueron: un modelo bifactorial, compuesto por dos factores correlacionados (enriquecimiento trabajo-familia y enriquecimiento familia-trabajo) y los 18 ítems iniciales como variables observables con sus respectivos errores; y un modelo tetrafactorial, conformado por cuatro factores correlacionados (correspondientes a las cuatro modalidades del enriquecimiento postuladas por Carslon: basado en el desarrollo, en la afectividad, en el capital y en la eficacia) y 18 ítems como indicadores observables, con sus respectivos errores de medición.

Modelo Bifactorial (dos factores con 18 items). Los resultados indicaron que este modelo no presentaba un adecuado grado de ajuste a los datos: SatorraBentler $\chi^{2}{ }_{(135)}=324,7, p<, 001$; CFI = ,850; RMSEA = ,08, IC 90\% [,07; ,09]. Los dos factores correlacionaron positiva y significativamente $(r=, 24, p<$ ,001). Las cargas factoriales estandarizadas $(p<, 05)$ en el factor enriquecimiento familia $\rightarrow$ trabajo se ubicaron entre ,30 y ,56, en tanto que las cargas correspondientes al factor enriquecimiento trabajo $\rightarrow$ familia se encontraron comprendidas entre ,43 y ,67. Se eliminaron del modelo seis ítems con bajas cargas factoriales estandarizadas, sin embargo, no se observó una mejoría en el grado de ajuste: Satorra-Bentler $\chi^{2}$ ${ }_{(107)}=318,2, p<, 001 ; \mathrm{CFI}=, 800$; RMSEA $=, 09$, IC 90\% [,07; ,10]. Los ítems eliminados en esta oportunidad coincidieron con los eliminados en el primer AFE ejecutado.
Modelo Tetrafactorial (cuatro factores: enriquecimiento basado en la afectividad, en el desarrollo, en el capital y en la eficacia; con 18 items). Los valores del grado de ajuste estuvieron alejados de los valores recomendados: SatorraBentler $\chi^{2}{ }_{(135)}=356,1, p<, 001$; CFI = ,886; RMSEA = ,09, IC 90\% [,08; ,10]. El factor enriquecimiento basado en la afectividad correlacionó positiva y significativamente con los factores de enriquecimiento basado en el desarrollo $(r=, 43, p<, 001)$ $\mathrm{y}$ enriquecimiento basado en la eficacia $(r$ $=, 32, p<, 001)$. El enriquecimiento basado en el capital presentó una correlación positiva y significativa con el enriquecimiento basado en el desarrollo ( $r$ $=, 68, p<, 001)$ y basado en la afectividad $(r=, 36, p<, 001)$. Las cargas factoriales estandarizadas $(p<, 05)$ en el factor enriquecimiento basado en la afectividad oscilaron entre ,29 y ,58, en el factor enriquecimiento basado en el desarrollo entre ,38 y ,56, en el factor enriquecimiento basado en la eficacia entre ,41 y , 72 y en el factor enriquecimiento basado en el capital entre ,41 y ,77.

Modelo Bifactorial (dos factores con 12 items). Los estadísticos de ajuste indicaron un aceptable grado de los mismos: Satorra-Bentler $\chi^{2}{ }_{(127)}=265,33, p<, 001$; $\mathrm{CFI}=, 912$; RMSEA = ,05, IC 90\% [,03; ,06]. Ambos factores correlacionaron positiva y significativamente $(r=, 49, p<$ ,001). Las cargas factoriales estandarizadas $(p<, 05)$ en el factor enriquecimiento familia $\rightarrow$ trabajo oscilaron entre ,49 y ,87, en tanto que las cargas correspondientes al factor enriquecimiento trabajo $\rightarrow$ familia se encontraron comprendidas entre ,63 y ,85.

Análisis de confiabilidad. Confirmada la estructura bifactorial de la escala, se examinó su confiabilidad mediante el cómputo de los coeficientes alfa ordinales. 
Para la escala completa se obtuvo un $\alpha$ ordinal $(, 76)$, ligeramente inferior de la que se obtuvo para cada dimensión (enriquecimiento trabajo $\rightarrow$ familia $\alpha$ ordinal $=$ , 81 , y enriquecimiento familia $\rightarrow$ trabajo $\alpha$ ordinal $=, 78$ ).

Análisis de validez. El coeficiente de validez convergente AVE alcanzó un valor de ,67 para la subescala enriquecimiento trabajo-familia y ,62 para la subescala familia trabajo. Tales valores constituyen indicadores de que los ítems elegidos para medirlas comparten más de la mitad de la varianza (Fornell \& Larcker, 1981) de cada una de las dos dimensiones que integran la EETF.

Análisis de la invarianza por sexo. A efectos de garantizar que la EETF pudiera ser empleada indistintamente con muestras de trabajadores varones o mujeres se determinó su invarianza factorial a nivel configural y métrico. Para explorar la invarianza configural se efectuó un AFC multigrupo por género que determinó que la estructura factorial era adecuada cuando ambos grupos se probaron juntos y sin restricciones $\left(\chi^{2} / g l=1,24, \mathrm{GFI}=, 90, \mathrm{CFI}\right.$ $=$,93, RMSEA = ,02). Para evaluar la invarianza métrica se realizó la prueba de diferencia de $\chi^{2}$, contrastando el $\chi^{2}$ obtenido en un primer modelo no constreñido con el $\chi^{2}$ correspondiente a un modelo alternativo con restricciones sobre los coeficientes de regresión. El monto de probabilidad asociado al estadístico de contaste no alcanzó valores estadísticamente significativos $\left(\mathrm{D} \chi^{2}=28,5 ; p=\right.$ $, 160)$, indicando entonces la no existencia de diferencias entre los grupos.

\section{Discusión}

El objetivo de este estudio fue adaptar y validar la Escala de Enriquecimiento Trabajo-Familia desarrollada por Carlson et al. (2006), para su empleo con trabajadores argentinos. Los análisis realizados indicaron que la escala adaptada posee adecuada validez y confiabilidad. Respecto de la validez estructural, los dos factores identificados a través del AFE y confirmados por el AFC, demostraron la naturaleza bidimensional del constructo. La estructura obtenida reproduce la solución encontrada tanto por los autores del instrumento (Carlson et al., 2006), como la informada en estudios de validación previos (Ghislieri et al., 2011; Lim et al., 2012; Tement et al., 2010; Vieira et al., 2014). Por lo que en sintonía con la configuración latente del constructo, se mantuvo la denominación propuesta por Carlson para cada dimensión: enriquecimiento trabajo $\rightarrow$ familia y enriquecimiento familia $\rightarrow$ trabajo, respectivamente. En este sentido, no hay que perder de vista que cuando Carlson y sus colaboradores describieron la bidireccionalidad del concepto, lo hicieron en términos de los recursos que el trabajo y la familia suministran a las personas para mejorar el rendimiento y la calidad de vida en ambos dominios. De allí que señalaran que el enriquecimiento puede provenir de experiencias positivas vividas, ya sea en el ámbito laboral, o en el familiar. Vale decir que las experiencias laborales pueden mejorar la calidad de vida en el dominio familiar por la seguridad, la confianza o la autorrealización que proporciona el trabajo (es decir, capital transferido desde el trabajo a la familia), por un estado emocional positivo (es decir, afectividad transferida desde el trabajo a la familia), o bien, por la adquisición de habilidades y conocimientos (es decir, desarrollo transferido desde el trabajo a la familia). Por su parte, las experiencias positivas y los recursos obtenidos en el dominio familiar pueden tener un impacto algo diferente sobre el ámbito laboral. Ya que, por ejemplo, el ámbito familiar puede mejorar 
el estado de ánimo o proporcionar nuevas habilidades (es decir, afectividad y desarrollo transferido desde la familia al trabajo) pero, además, puede proporcionar un sentido de cuidado, dedicación y premura en la atención de los problemas que mejorarían la concentración en el trabajo (es decir, eficiencia transferida desde la familia al trabajo).

Por tal motivo, la tendencia dominante en la literatura referida a trabajo-familia es la de considerar que ambas dimensiones del enriquecimiento son empíricamente diferentes y tienen un patrón de dominio específico (Byron, 2005). Esto significa que los predictores provienen del dominio de origen y las consecuencias se hacen sentir en el dominio receptor. Esta perspectiva se ha visto fortalecida a partir de la postulación de la teoría de los recursos ganados (Wayne et al., 2007), la que es consistente con el modelo del dominio específico en la generación del proceso de enriquecimiento trabajo-familia. Desde esta óptica, los factores clave de crecimiento y desarrollo (y, por consiguiente también el enriquecimiento trabajo-familia) son recursos personales y medio ambientales del dominio de origen. Por lo tanto, como el componente central de los recursos surge del dominio de origen, el apoyo basado en el trabajo es considerado un motor afectivo e instrumental esencial en el proceso de enriquecimiento trabajo-familia (Bakker \& Demerouti, 2007), porque fomenta el crecimiento, el aprendizaje y el desarrollo de los empleados, lo que aumenta la calidad de vida familiar, genera afectos positivos y facilita el logro de los objetivos, y viceversa.

Los resultados obtenidos indicaron que la escala posee una buena validez convergente, vale decir que la varianza del constructo enriquecimiento trabajo-familia se puede explicar adecuadamente a través de los indicadores elegidos. Asimismo, los resultados mostraron que la escala posee una aceptable consistencia interna, comparable a la de la versión original y se sitúa dentro del rango obtenido en otras investigaciones en las que se ha empleado este instrumento (Lim et al., 2012; Tement et al., 2010). Como el coeficiente alfa ordinal para cada dimensión fue mayor que el coeficiente para la escala total, con finalidades prácticas podrían utilizarse las subescalas por separado, ya que poseen una confiabilidad aceptable. Asimismo, se analizó y verificó la invarianza conceptual y métrica del instrumento en función del sexo. Los resultados indicaron que no hay diferencias de género, por lo que la EETF podría ser utilizada con la misma validez y confiabilidad tanto con muestras de trabajadores varones como con muestras de trabajadoras mujeres.

Fortalezas, limitaciones e implicancias prácticas

A pesar de los auspiciosos resultados obtenidos, es necesario efectuar algunas consideraciones en términos de la validez externa del estudio. En primer lugar, la estabilidad de las dimensiones a lo largo del tiempo no ha sido probada, por lo que en estudios futuros sería recomendable que se investigue su confiabilidad testretest. En segundo lugar, la naturaleza transversal del estudio impide establecer relaciones causales, por lo que sería pertinente realizar estudios longitudinales, así como complementar las medidas autodescriptivas empleadas con otros métodos de evaluación como, por ejemplo, entrevistas (tanto con el trabajador como con otros miembros de la familia) o indicadores psicofisiológicos. Finalmente, hay que señalar que por tratarse de una medida autodescriptiva, la escala evalúa el ETF percibido, de modo que las respuestas 
podrían verse contaminadas por el componente subjetivo que esto conlleva, limitando la generalización de los resultados.

Sin embargo, a pesar de tales limitaciones, hay que destacar que la versión validada de la EETF, debido a que es una medida integrada por pocos ítems, resulta muy parsimoniosa y práctica de administrar, ya que es sabido que los instrumentos largos requieren más tiempo para ser completados, presentan mayores porcentajes de datos faltantes y suelen generar mayores tasas de rechazo (Russell et al., 2004). Desde un punto de vista práctico, es importante disponer de estas escalas breves en el idioma del encuestado, para que permitan conocer rápidamente de qué manera los trabajadores viven la relación entre los asuntos familiares y laborales. La versión validada de la EETF puede ser útil para investigadores y profesionales, tanto del ámbito familiar como organizacional y sobre todo para los que buscan un instrumento simple, válido y confiable a efectos de diagnosticar los inhibidores y los facilitadores del enriquecimiento entre el trabajo y la familia. A partir del conocimiento de tales circunstancias se podrán diseñar estrategias e instrumentar acciones que favorezcan una mejor interrelación entre ambas esferas vitales lo que, indudablemente, repercutirá en una mejor calidad de vida para todos.

\section{Conclusiones}

En síntesis, los resultados encontrados indican que la adaptación argentina de la EETF posee propiedades psicométricas satisfactorias, por lo que resulta ser una medida válida y confiable para la evaluación del enriquecimiento entre el trabajo y la familia. La EETF adaptada para su empleo con trabajadores hispanoparlantes quedó configurada como un modelo bidimensional, con los factores rotulados como enriquecimiento trabajo $\rightarrow$ familia y enriquecimiento familia $\rightarrow$ trabajo. Este modelo, similar al propuesto por Carlson et al. (2006), fue confirmado con una bondad de ajuste aceptable y mostró invarianza métrica y configural en mujeres y varones. Los dos componentes del constructo presentaron una buena consistencia interna, por lo que con fines prácticos, podrían aplicarse individualmente.

\section{Referencias}

Allis, P. \& O'Driscoll, M. (2008). Positive effects of nonwork-to-work facilitation on well-being in work, family and personal domains. Journal of Managerial Psychology, 23(3), 273-291. http://dx.doi.org/10.1108/02683940810861383

Bagozzi, R. P. \& Yi, Y. (1988). On the evaluation of structural equation models. Journal of the Academy of Marketing Science, 16(1), 74-94.

http://dx.doi.org/10.1007/bf02723327

Bakker, A. \& Demerouti, E. (2007). The job demands-resources model: State of the art. Journal of Managerial Psychology, 22(3), 309-328. http://dx.doi.org/10.1108/02683940710733115

Bentler, P. (2006). EQS 6 structural equations program manual. Encino: Multivariate Software. http://dx.doi.org/10.4324/9780203726532 
Byron, K. (2005). A meta-analytic review of the work-family conflict and its antecedents. Journal of Vocational Behavior, 67(2), 169-198.

http://dx.doi.org/10.1016/j.jvb.2004.08.009

Carlson, D. S., Ferguson, M., Kacmar, K., Grzywacz, J. G., \& Whitten, D. (2011). Pay it forward: The positive crossover effects of supervisor work-family enrichment. Journal of Management, 37(3), 770-789.

http://dx.doi.org/10.1177/0149206310363613

Carlson, D. S., Kacmar, K. M., Wayne, J. H., \& Grzywacz, J. G. (2006). Measuring the positive side of the work-family interface: Development and validation of a work-family enrichment scale. Journal of Vocational Behavior, 68(1), 131-164.

http://dx.doi.org/10.1016/j.jvb.2005.02.002

Cattell, R. B. (1966). The scree test for the number of factors. Multivariate Behavioral Research, 1(2), 245-276.

http://dx.doi.org/ 10.1207/s15327906mbr0102_10

Demerouti, E., Peeters, M. W., \& van der Heijden, B. M. (2012). Work-family interface from a life and career stage perspective: The role of demands and resources. International Journal of Psychology, 47(4), 241-258.

http://dx.doi.org/10.1080/00207594.2012.699055

Dimitrov, D. M. (2010). Testing for factorial invariance in the context of construct validation. Measurement and Evaluation in Counseling and Development, 43(2), 121-149. http://dx.doi.org/10.1177/0748175610373459

Fornell, C. \& Larcker, D. F. (1981). Evaluating structural equations models with unobservable variables and measurement error. Journal of Marketing Research, 18(1), 39-50. http://dx.doi.org/10.2307/3151312

Frone, M. R. (2003). Work-family balance. En J. C. Quick \& L. E. Tetrick (Eds.), Handbook of Occupational Health Psychology (pp. 143-162). Washington, DC.: American Psychological Association. http://dx.doi.org/10.1037/10474-007

Gadermann, A. M., Guhn, M., \& Zumbo, D. (2012). Estimating ordinal reliability for Likert-type and ordinal item response data: A conceptual, empirical, and practical guide. Practical Assessment, Research \& Evaluation, 17(3), 1-13. Recuperado de http://pareonline.net/pdf/v17n3.pdf

George, D. \& Mallery, M. (2011). SPSS for Windows step by step: A simple guide and reference 18.0 Update. Boston: Allyn \& Bacon.

Ghislieri, C., Martini, M., Gatti, P., \& Colombo, L. (2011). The "bright side" of the workfamily interface: A brief work-family enrichment scale in a sample of health professionals. TPM-Testing, Psychometrics, Methodology in Applied Psychology, 18(4), 211-230. Recuperado de http://www.tpmap.org/wp-content/uploads/2014/11/18.4.2.pdf

Greenhaus, J. H. \& Powell, G. N. (2006). When work and family are allies: A theory of work-family enrichment. The Academy of Management Review, 31(1), 72-92. http://dx.doi.org/10.5465/AMR.2006.19379625 
Grzywacz, J. G. \& Marks, N. F. (2000). Reconceptualizing the work-family interface: An ecological perspective on the correlates of positive and negative spillover between work and family. Journal of Occupational Health Psychology, 5(1), 111-126.

http://dx.doi.org/10.1037/1076-8998.5.1.111

Haar, J. M. \& Bardoel, E. A. (2008). Positive spillover from the work-family interface: A study of Australian employees. Asia Pacific Journal of Human Resources, 46(3), 275-287. http://dx.doi.org/10.1177/1038411108095759.

Hair, J. F., Black, W. C., Babin, B. J., Anderson, R. E., \& Tatham, R. L. (2006). Multivariate data analysis. New Jersey: Pearson.

Hakanen, J. J., Peeters, M. C., \& Perhoniemi, R. (2011). Enrichment processes and gain spirals at work and at home: A 3-year cross-lagged panel study. Journal of Occupational and Organizational Psychology, 84(1), 8-30.

http://dx.doi.org/10.1111/j.2044-8325.2010.02014.x

Hanson, G. C., Hammer, L. B., \& Colton, C. L. (2006). Development and validation of a multidimensional scale of perceived work-family positive spillover. Journal of Occupational Health Psychology, 11(3), 249-265.

http://dx.doi.org/10.1037/1076-8998.11.3.249

Herdman, M., Fox-Rushby, J., \& Badía, X. (1998). A model of equivalence in the cultural adaptation of HRQOL instruments: The universalist approach. Quality of Life Research, 7(4), 323-335.

http://dx.doi.org/10.1023/a:1024985930536

Hinkin, T. R. (1998). A brief tutorial on the development of measures for use in survey questionnaires. Organizational Research Methods, 2(1), 104-121.

http://dx.doi.org/10.1177/109442819800100106

Hu, L. T. \& Bentler, P. M. (1999). Cutoff criteria for fit indexes in covariance structure analysis: Conventional criteria versus new alternatives. Structural Equation Modeling, 6(1), 1-55.

http://dx.doi.org/10.1080/10705519909540118

Lim, D., Choi, M., \& Song, J. (2012). Work-family enrichment in Korea: Construct validation and status. Leadership \& Organization Development Journal, 33(3), 282-299. http://dx.doi.org/10.1108/01437731211216470

Lloret, S., Ferreres, A., Hernández, A., \& Tomás, I. (2014). El análisis factorial exploratorio de los ítems: una guía práctica, revisada y actualizada. Anales de Psicología, 30(3), 1151-1169.

http://dx.doi.org/10.6018/analesps.30.3.199361

Luthans, F. \& Youssef, C. M. (2007). Emerging positive organizational behavior. Journal of Management, 33(3), 321-349.

http://dx.doi.org/10.1177/0149206307300814

Marks, S. R. (1977). Multiple roles and role strain: Some notes on human energy time and commitment. American Sociological Review, 42(6), 921-936.

http://dx.doi.org/10.2307/2094577 
Masuda, A. D., McNall, L. A., Allen, T. D., \& Nicklin, J. M. (2012). Examining the constructs of work-to-family enrichment and positive spillover. Journal of Vocational Behavior, 80(1), 197-210.

http://dx.doi.org/10.1016/j.jvb.2011.06.002

McNall, L. A., Nicklin, J. M., \& Masuda, A. D. (2010). A meta-analytic review of the consequences associated with work-family enrichment. Journal of Business and Psychology, 25(3), 381-396.

http://dx.doi.org/10.1007/s10869-009-9141-1

Meeuwisse, M., Born, M. P., \& Severiens, S. E. (2011). The family-study interface and academic outcomes: Testing a structural model. Journal of Educational Psychology, 103(4), 982-990.

http://dx.doi.org/10.1037/a0024420

Miles, J. \& Shevlin, M. (2001). Applying regression and correlation: A guide for students and researchers. London: Sage Publications.

Montero, I. \& León, O. (2007). A guide for naming research studies in Psychology. International Journal of Clinical and Health Psychology, 7(3), 847-862. Recuperado de http://www.aepc.es/ijchp/GNEIP07_es.pdf

Odle-Dusseau, H. N., Britt, T. W., \& Greene-Shortridge, T. M. (2012). Organizational workfamily resources as predictors of job performance and attitudes: The process of work-family conflict and enrichment. Journal of Occupational Health Psychology, 17(1), 28-40. http://dx.doi.org/10.1037/a0026428

Poelmans, S. A., O’Driscoll, M., \& Beham, B. (2005). A review of international research in the field of work and family. En S. A. Poelmans (Ed.), Work and family: An international research perspective (pp. 3-46). Mahwah: Erlbaum.

Russell, S. S., Spitzmuller, C., Lin, L. F., Stanton, J. M., Smith, P. C., \& Ironson, G. H. (2004). Shorter can also be better: The abridged job in general scale. Educational and Psychological Measurement, 64(5), 878-893.

http://dx.doi.org/10.1177/0013164404264841

Satorra, A. (2002). Asymptotic robustness in multiple group linear-latent variable models. Econometric Theory, 18(2), 297-312.

http://dx.doi.org/10.1017/S0266466602182041

Shah, R. \& Goldstein, S. (2006). Use of structural equation modeling in operations management research: Looking back and forward. Journal of Operations Management, 24(2), 148-169. http://dx.doi.org/10.1016/j.jom.2005.05.001

Tajfel, H. \& Turner, J. C. (2004). The social of identity theory of intergroup behaviour. En S. Worchel \& W. G. Austin (Eds.), Psychology of intergroup relations (pp. 7-24). Chicago: Nelson.

Tement, S., Korunka, C., \& Pfifer, A. (2010). Toward the assessment of the work-family interface: Validation of the Slovenian versions of work-family conflict and work-family enrichment scales. Horizons of Psychology, 19(3), 53-74.

http://dx.doi.org/10.1037/t41572-000 
van Steenbergen, E. \& Ellemers, N. (2009). Is managing the work-family interface worthwhile? Benefits for employee health and performance. Journal of Organizational Behavior, 30(5), 617-642.

http://dx.doi.org/10.1002/job.569

Vieira, J. M., Lopez, F. G., \& Matos, P. M. (2014). Further validation of work-family conflict and work-family enrichment scales among Portuguese working parents. Journal of Career Assessment, 22(2), 329-344.

http://dx.doi.org/10.1177/1069072713493987

Wayne, J. H., Grzywacz, J. G., Carlson, D. S., \& Kacmar, K. M. (2007). Work-family facilitation: A theoretical explanation and model of primary antecedents and consequences. Human Resource Management Review, 17(1), 63-76.

http://dx.doi.org/10.1016/j.hrmr.2007.01.002

Wayne, J. H., Musisca, N., \& Fleeson, W. (2004). Considering the role of personality in the work-family experience: Relationships of the Big Five to work-family conflict and facilitation. Journal of Vocational Behavior, 64(1), 108-130.

http://dx.doi.org/10.1016/S0001-8791(03)00035-6

Zumbo, D. \& Oliden, P. (2008). Coeficientes de fiabilidad para escalas de respuesta categórica ordenada. Psicothema, 20(4), 896-901. Recuperado de http://www.psicothema.com/pdf/3572.pdf 